\title{
Colorimetric Determination of Formaldehyde Using 1,3-Diphenyl-2-thiohydantoin and Sodium Hydroxide
}

\author{
Miyuki Takayanagi*, Yasuo Morishima*, Shoji Goto*, Iwazo Hasegawa*, Tsuneo Fukuda*, \\ Masashi USAMI**, Toshiko NAITo** and Tamotsu YASHIRo** \\ *Aichi Prefecture Red Cross Blood Center, San-nomaru, Naka-ku, Nagoya 460 \\ **Faculty of Pharmaceutical Sciences, Nagoya City University, \\ Tanabe-dori, Mizuho-ku, Nagoya 467
}

\begin{abstract}
A method for the determination of formaldehyde concentration in water is described. It is based on a coupling reaction of 1,3-diphenyl-2-thiohydantoin with formaldehyde. In this reaction an anionic pigment is formed by addition of sodium hydroxide solution. The color intensity and formaldehyde concentrations were linearly related in the range up too $100 \mu \mathrm{M}$. The minimum detectable concentration was $2 \mu \mathrm{M}$. The precision (R.S.D.) was 3.62\%.
\end{abstract}

Keywords Formaldehyde, 1,3-diphenyl-2-thiohydantoin, anionic pigment, spectrophotometry

Several methods for determination and estimation of formaldehyde in water have been reported. They involve simple, sensitive, and reproducible methods such as spectrophotometric methods ${ }^{1-11}$, gas liquid chromatographic methods ${ }^{12,13}$, and others. ${ }^{14,15}$ However, these methods exhibit various disadvantages with regard to sensitivity, detectable range, and troublesome operation.

2-Thiobarbituric acid (TBA) has been widely used for the determination of malonaldehyde (MA) ${ }^{16-18}$ produced from lipid peroxide in biological materials. TBA is reacted with carbonyl compounds, in the presence of catalysts such as alkalis and acids, to produce the coupled and colored compounds. ${ }^{19-21} 1,3-$ Diphenyl-2-thiohydantoin (DPTH) has a molecular structure similar to TBA, and is reactive also with aldehydes.

We have tested the reaction of DPTH with formaldehyde and sodium hydroxide, and here report a method for the determination of formaldehyde with DPTH and sodium hydroxide.

\section{Experimental}

\section{Materials}

DPTH was synthesized according to the method described by Shirai et al. ${ }^{22}$ Formaline was purchased from Nakakita Pharmaceutical Co., Nagoya, Japan; other chemicals were from Wako Pure Chemical Industries, Osaka, Japan.

DPTH solution was prepared by dissolving $600 \mathrm{mg}$ of DPTH in $100 \mathrm{ml}$ of acetone. Standard solutions of formaldehyde were prepared by diluting formaline with water to give formaldehyde concentrations ranging from 2 to $100 \mu \mathrm{M}\left(\mathrm{M}=\mathrm{mol} \mathrm{dm}^{-3}\right)$, as determined by the iodine method. ${ }^{23}$

\section{Procedure for determination of formaldehyde}

DPTH solution, $5 \mathrm{ml}$, was pipetted into test tubes. Formaldehyde standards, samples, or water as a blank, each $0.5 \mathrm{ml}$, and $1 \mathrm{M}$ sodium hydroxide solution, 0.1 $\mathrm{ml}$, were pipetted into each test tube and mixed well. The filled test tubes were incubated at room temperature for $30 \mathrm{~min}$. The absorbances of these solutions were measured at $535 \mathrm{~nm}$ against the blank (Fig. 1).

Formaldehyde was determined by the 3-methyl-2benzothiazolinone hydrazone method $^{7}$ (the MBTH method), as previously described.

\section{Results}

Effect of reagent concentrations on extent of color formation

The color formation has been found to be optimum at a concentration of acetone solution containing 22 mM DPTH. Under the above conditions, the reacting solution contained DPTH in a molar ratio more than 2200 times that of formaldehyde. When volumes of the samples were tested between 0.2 and $4 \mathrm{ml}$, the reaction solution was turbid at a volume of under $0.3 \mathrm{ml}$. Thus the sample volume was set at $0.5 \mathrm{ml}$.

\section{Reaction with other compounds}

The effects of other compounds such as acetal, propionaldehyde, glutaraldehyde, malonaldehyde, dex- 


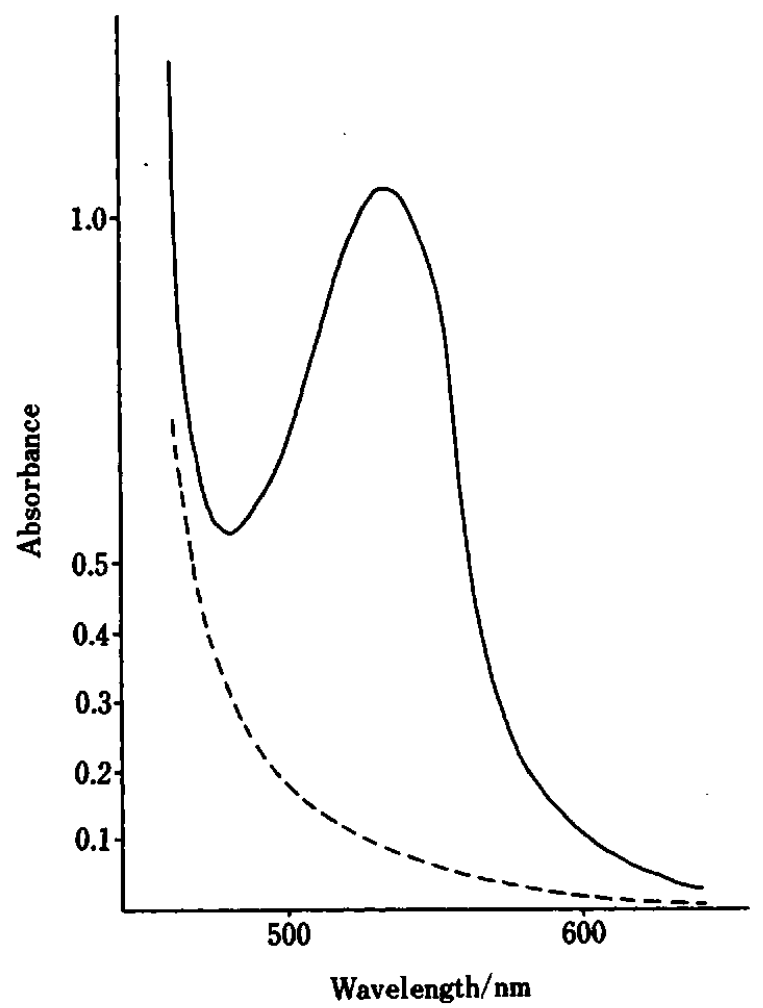

Fig. 1 Visible absorption spectra. Formaldehyde concentrations in samples, $0(--)$ and $78.6(-) \mu \mathrm{M}$.

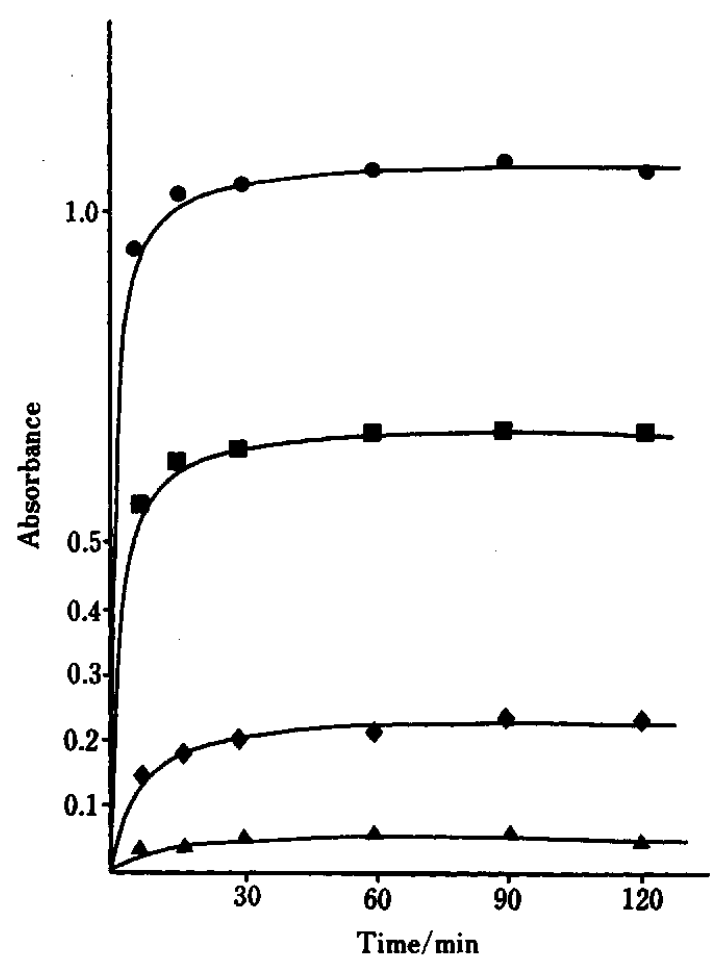

Fig. 2 Time-course of color reaction. Formaldehyde concentrations in samples, $0(\Delta), 19.6(\bullet), 49.1(\square)$, and $78.6(-) \mu \mathrm{M}$.

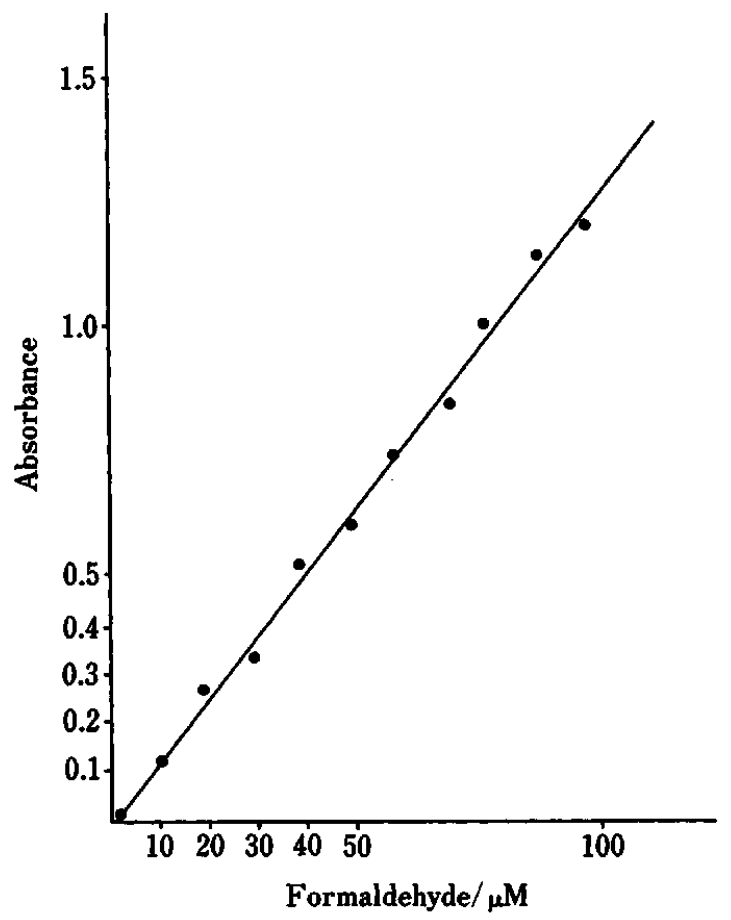

Fig. 3 A typical calibration curve for formaldehyde by the proposed method. $n=11, r=0.998$, slope $=0.0127$, intercept $=-\mathbf{0 . 0 0 6 9}$.

trose, lactose, levulose, and maltose on colorimetry, were tested for the proposed method. Pigments produced from these compounds with DPTH were only under 0.01 absorbance unit at concentrations of these compounds about $10^{4}$ times those of formaldehyde samples.

\section{Time-course variation of color reaction}

Figure 2 illustrates the time-course variation of color reaction of DPTH with formaldehyde and sodium hydroxide. The reaction was nearly completed within $30 \mathrm{~min}$. Although a certain degree of variation in absorbance occurs in the absence of the sample, it does not interfere with linearity, because the absorbance of the blank can be subtracted from that of each sample.

\section{Sensitivity and precision}

As shown Fig. 3, good linearity was obtained between absorbances and formaldehyde concentrations (up to $100 \mu \mathrm{M}$ ). The lower limit of the concentration was $2 \mu \mathrm{M}$. Table 1 gives the within-run results for each concentration in 10 test tubes.

\section{Comparison of methods}

Figure 4 shows the formaldehyde concentrations of the identical samples measured by the proposed method and the MBTH method simultaneously. A good correlation was observed for the results of these two methods. 
Table 1 Precision of the proposed method for the determination of formaldehyde

\begin{tabular}{|c|c|c|c|}
\hline \multicolumn{3}{|c|}{ Formaldehyde/ $\mu \mathrm{M}$} & \multirow{3}{*}{ R.S.D. } \\
\hline \multirow[t]{2}{*}{ Added } & \multicolumn{2}{|c|}{ Measured } & \\
\hline & Mean & S.D. & \\
\hline \multicolumn{4}{|c|}{ Within-run $(n=10)$} \\
\hline $\begin{array}{r}9.8 \\
39.3 \\
78.6\end{array}$ & $\begin{array}{l}10.3 \\
38.8 \\
74.4\end{array}$ & $\begin{array}{l}0.24 \\
0.65 \\
1.76\end{array}$ & $\begin{array}{l}2.31 \\
1.67 \\
2.36\end{array}$ \\
\hline \multicolumn{4}{|c|}{ Between-run $(n=10)$} \\
\hline 39.3 & 38.3 & 1.39 & 3.62 \\
\hline
\end{tabular}

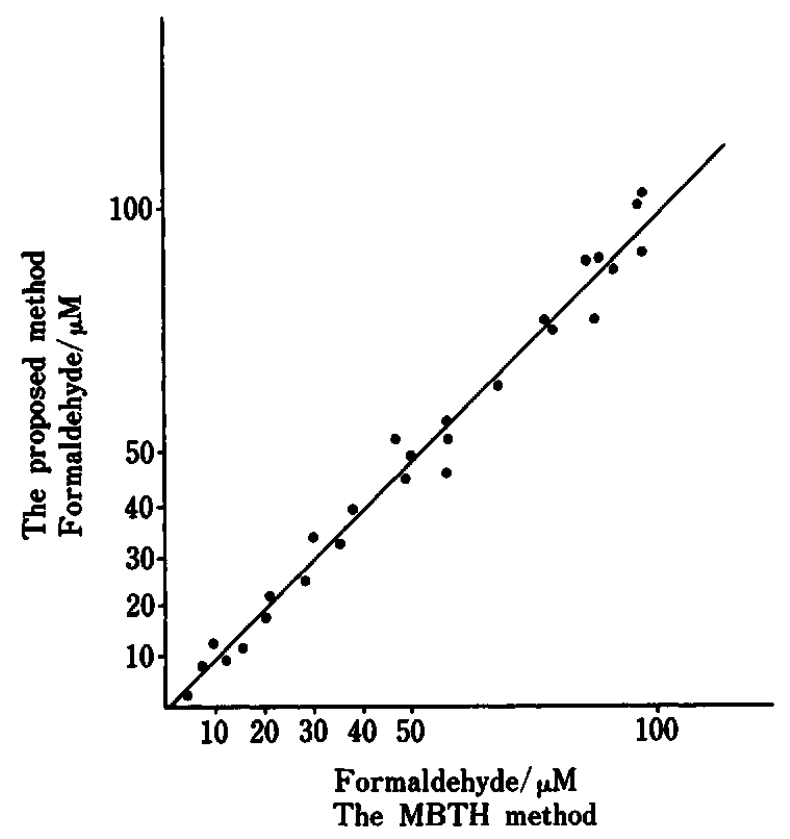

Fig. 4 Correlation between the proposed method $(y)$ and the MBTH method $(x) . \quad n=27, r=0.994$, slope $=$ 1.018 , intercept $=-0.193, \bar{x}=52.64, \bar{y}=53.41$.

\section{Discussion}

The product of the color reaction among DPTH, formaldehyde, and sodium hydroxide was found to be a red pigment. In the color reaction of TBA with MA, Sinnhuber et al..$^{24}$ showed that two molecules of TBA was coupled with one molecule of MA to produce a pigment. Similarly, in the reaction among DPTH, formaldehyde, and sodium hydroxide, we presumed that the structure of the red pigment produced in the proposed method was similar to that of the coupling compound of TBA with MA.

The method for the colorimetric determination of formaldehyde is sensitive, reproducible, and simple. The red pigment which resulted from DPTH, formaldehyde, and sodium hydroxide was sufficiently stable to allow reasonable precise measurement of formal- dehyde. The absorbance varied very little within about 60 min after incubation at room temperature for 30 $\min$.

A high degree of accuracy is shown, thanks to the good correlation between the proposed method and the MBTH method (Fig. 4).

Several methods for spectrophotometric determination of formaldehyde have been reported: for example, the acetylacetone method ${ }^{1,2}$, the phenylhydrazine $\operatorname{method}^{3}$, the pararosaniline method ${ }^{4,5}$, the MBTH method $^{6-8}$, the chromotropic acid method $^{9}$, and others. ${ }^{10,11}$ Among them, the most sensitive method is the MBTH method, which is affected by other aldehydes such as acetaldehyde, propionaldehyde, crotonaldehyde, and others. ${ }^{7}$ On the other hand, the sensitivity of the proposed method was almost similar to that of the MBTH method ${ }^{7}$ and the proposed method was affected neither by other aldehydes such as acetal, propionaldehyde, glutaraldehyde, and MA nor by aldoses such as dextrose, lactose, levulose, and maltose. Therefore, the proposed method is considered to be very useful for determining the concentrations of formaldehyde.

\section{References}

1. T. Nash, Biochem. J., 55, 416 (1953).

2. J. Cochin and J. Axelrod, J. Pharmacol. Exp. Therap., 125, 105 (1959).

3. R. W. Kersey, J. R. Moddocks and T. E. Johnson, Analyst [London], 65, 203 (1940).

4. G. R. Lyles, F. B. Dowling, and V. J. Blanchard, J. Air. Poll. Cont. Ass., 15, 106 (1965).

5. A. C. Rayner and C. M. Jephcott, Anal. Chem., 33, 627 (1961).

6. T. R. Hauser and R. L. Cummins, Anal. Chem., 36, 679 (1964).

7. E. Sawicki, T. R. Hauser, T. W. Stanley and W. Elbert, Anal. Chem., 33, 93 (1961).

8. N. Tajima and Y. Kurosaki, Bunseki Kagaku, 23, 464 (1974).

9. S. T. Cuffe, Arch. Environ. Health., 6, 422 (1963).

10. J. Chrastil and J. T. Wilson, Anal. Biochem., 63, 202 (1975).

11. J. M. Munson and T. G. Hodgkins, J. Pharm. Sci., 64, 1043 (1975).

12. T. R. Bosin, B. Holmstedt, A. Lundman and O. Beck, Anal. Biochem., 128, 287 (1983).

13. C. Improta, G. Nota, C. Ferretti and U. Papale, J. Chromatogr., 285, 385 (1984).

14. W. M. Grant, Anal. Chem., 20, 267 (1948).

15. M. J. Fletcher, Clin. Chim. Acta, 22, 393 (1968).

16. K. Nakashima, T. Ando, K. Nakamura and S. Akiyama, Chem. Pharm. Bull., 31, 2523 (1983).

17. P. P. Bird, Silas S. O. Hung, M. Hadley, and H. H Draper, Anal. Biochem., 128, 240 (1983).

18. K. Yagi, I. Nishigaki and H. Ohama, Vitamine (in Japanese), 37, 105 (1968)

19. A. W. Dox and G. P. Plaisance, J. Am. Chem. Soc., 38, $2164(1916)$ 
20. H. I. Kohn and M. Liversedge, J. Pharmacol., 82, 292 (1944).

21. S. Patton, M. Keeney and G. W. Kurtz, J. Am. Oil Chemists'Soc., 28, 391 (1951).

22. H. Shirai, T. Yashiro and I. Miwa, Ann. Rep. Pharm. Nagoya City Univ., 14, 63 (1966).

23. M. Ishidate, Y. Nagase, I. Kawashiro, T. Shimomura and T. Uno, "The Explanation of The Japan Pharmaco- poeia" Tenth Ed., p. D-845, Hirokawa, Tokyo, (1981).

24. R. O. Sinnhuber, T. C. Yu and T. H. Yu, Food Res., 23, 626 (1958).
(Received December 21, 1984) (Accepted March 27, 1985) 